\title{
Circuit Breaker Screw Mounting MachineBased on Visual Control
}

\author{
Keping Zhang ${ }^{1}$ Xiangyang Du ${ }^{2}$ Yingbo Geng ${ }^{2}$ \\ (School Of Mechanical Engineering,Shanghai University Of Engineering Science, China,Shanghai,LongTeng \\ Road 333,201620)
}

\begin{abstract}
Due to the precise positioning of the screw housing of the plastic housing circuit breaker and the correct position and attitude adjustment, it is generally necessary that the mechanical positioning can not meet the requirements of the simplified mechanism and flexible installation. Using the industrial CCD camera plastic shell circuit breaker on the screw position information, and then use the open source computer vision library software for image processing to obtain the screw position information through the SPI bus to the SM32 chip for processing information to drive the corresponding servo to the screw installation. By comparing the experimental data, we found that work efficiency of machine installation is about 13 times to that of the ordinary installation process.
\end{abstract}

Key Word: Plastic case circuit breaker, Screw mounting, STM32 control card, CCD camera

\section{INTRODUCTION}

The extensive use of plastic shell circuit breakers makes it very important in the production and installation industry.The precise positioning of plastic shell circuit breakers is an important part in the assembly industry, because the intelligent rapid tracking and positioning has become a trend in the assembly industry.The project first utilizes the industrial CCD camera to shoot the image information of the screw hole on the plastic shell circuit breaker, and then take advantage of the visual acquisition module to process the image for the obtaining of the precise position of the screw installation.In this paper, a precise positioning system of plastic shell circuit breaker is proposed and its composition and principle are analyzed as well.The hardware platform includes hardware design, selection, placement of industrial CCD camera, motion control and image acquisition module design.The core part of the software plastic shell breaker adopts modular design.The core software can be divided into six modules: image acquisition module, GUI module, camera calibration, motion control, I/O control module and emergency interrupt module.The six modules work together, according to the characteristics of the image to find the corresponding location, drive the screw holder to complete the installation of screws.At the same time, in order to make the interface more human-computer interaction, the dynamic position of the screw and the plastic shell circuit breaker to be installed is displayed on the position of real-time display.

\section{INSTITUTIONAL OPERATIONS SECTION}

The structure of plastic case circuit breaker requires a flexible screw mounting machine to complete the circuit breaker assembly.The project uses stepper motor as the driver of the plastic shell breaker screw mounting machine, driving speed reducer of screw mounting machine[1].Screw mounting reducer part is composed of a large gear and a small gear reducer, which works together to complete the matching process, wherein the pinion completes the rotation adjustment component of the screwdriver, and that the large gear drives screw is used to realize the linear upward feed movement of the screw.The screws used in the plastic shell circuit breaker screw mounting machine are $\mathrm{M} 3 \mathrm{X} 0.7 \mathrm{~mm}$ and $\mathrm{M} 6 \mathrm{X} 10 \mathrm{~mm}$ respectively, not only the matching of the screws is completed, but also the matching of the speed and the feed.In order to meet these requirements, we designed a variable screwdriver and an electromagnetic clutch to match different pitchWhen the screws are fitted to the three metal pieces of the plastic housing circuit breaker, the metal sheet is deformed and the screw mounting becomes difficult.In order to solve this problem, it is necessary to design a screw and nut to install the locking mechanism to ensure that the screw can be successfully installed on the plastic shell protector.During the installation of the screw, it is important to track the trajectory of the screw[2], in this paper, the movable adjustment screw nut locking mechanism is designed for the deformation of metal shell of plastic shell protector. 


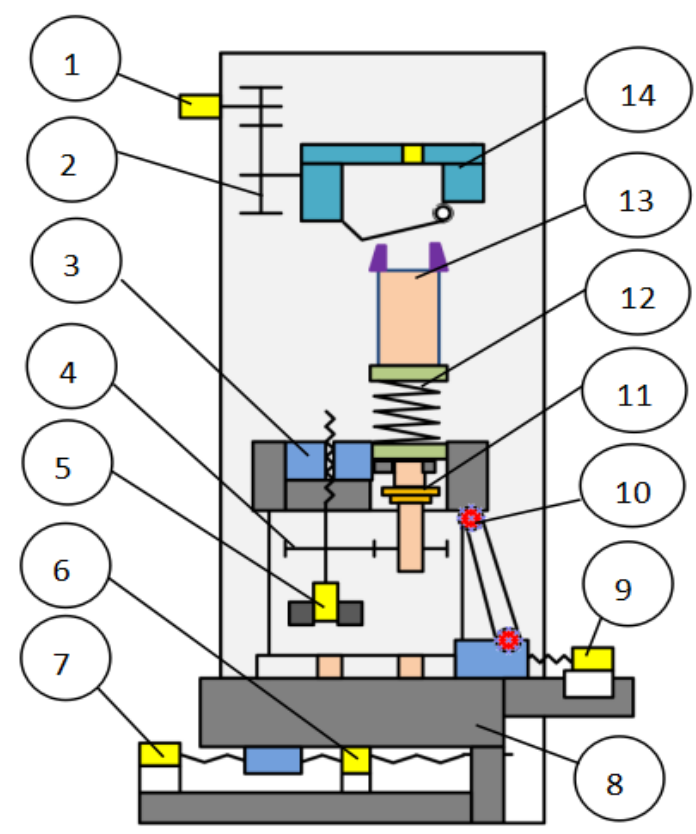

1 adjust motor 1; 2 reducer gears 1; 3 lift nuts; 4 reducer gears2; 5 screw mounting motor;

6 platform Y-axis motor; 7 platform X-axis motor; 8 frame; 9 attitude adjustment motor;

10 attitude adjustment lever; 11 electromagnetic clutch; 12 flexible mechanism; 13 adjustable screwdriver and locking mechanism; 14 circuit breaker fixture

Figure 1 transmission structure diagram

Molded Case Circuit Breaker Screw mounting machine requires not only the design of the mechanism, but also the need of accurate positioning in three-dimensional space.Through the industrial CCD camera, shooting screw and screw tip of the bottom and front, to capture the three-dimensional coordinate information of the screw hole and the screw head,and sent them to the STM32 motion controller for information processing. The STM32 motion controller transfers the processed information to the stepper motor that drives the X, Y, Z axes to complete the adjustment and positioning of the screw and screwdriver.After positioning, start the screwdriver to rotate and feed the stepper motor to complete the installation of the screw.In the light of the signals transmit to the motion control card torque sensor and CCD camera,determine whether the degree of tightening of the screw and the installation position and attitude deviation meets the requirements. The STM32 motion control board's LEDs help us determine if the next screw is going to be installed.

\section{Control system}

The control system of plastic shell circuit breaker consists of eight parts: fill light system, torque signal acquisition module, image acquisition module, display panel, STM32 motion controller, indicator module, switch module and stepper motor drive module. light system is to increase the brightness in the dark place of the mechanism, so that more complete and clear pattern could be collected.The torque signal acquisition module converts the torque analog information of the screwdriver to digital information that STM32 motion control card can accept.The image acquisition module processes the captured image into three-dimensional coordinate and the positional attitude of the screwdriver. The SPI bus is used to transfer the coordinates and position and attitude information to the STM32 motion control board for the system. The image acquisition module embeds the target locking function program for image processing, and extracts the coordinates of the corresponding points. In order to improve the human-computer interaction experience, the data that the user designed, the coordinates of the screwdriver' position, the condition of the running system are all displayed in the panel.STM32 controller's function is accomplished by the master chip STM32F103ZEY7A. The indicator module of plastic shell circuit breaker module is mainly to show the failure and emergency treatment of the situation.Depending on the combination of three LEDs to determine the appropriate failure or emergency information, there are nine different states in all[3]. The switch module is a manual operation with an emergency stop button. The effect of stepping motor driving module is to shift STM32 stepper motor control pulse signal to the rated voltage or rated current pulses that in line with stepping motor. 


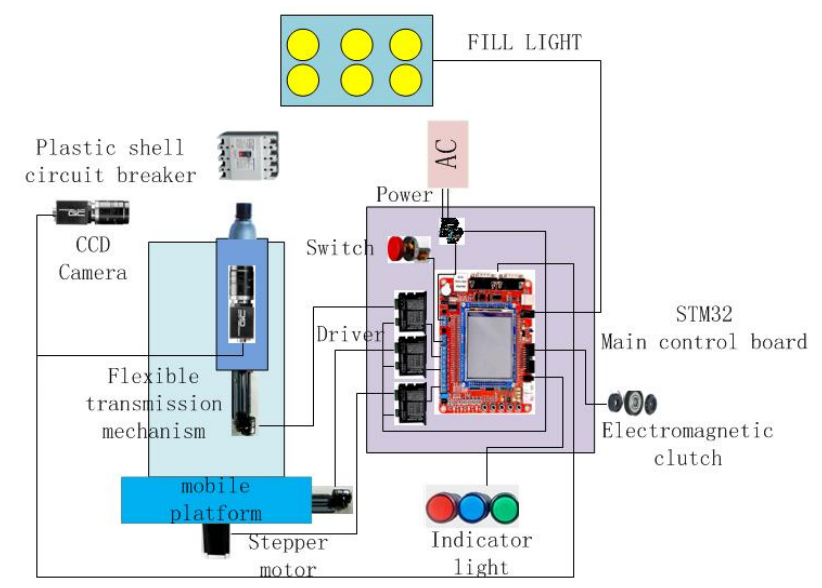

Figure 2 control system

The whole cause is performed as follows: Firstly, the position and position of each screw hole, captured by the CCD camera, is processed by the image processing module to determine the screw and screw tip coordinates and position posture of the plastic shell circuit breaker, and then, after the image processing, the coordinates (x, $\mathrm{y}, \mathrm{z}$ ) of the screw and screwdriver are transmitted to the STM32 motion control board via the SPI bus. Finally, the stepper motor is driven by the signals that processed through motion control board to install the screws.

\section{Experimental demonstration}

The efficiency and quality verification of plastic shell circuit breaker screw mounting machine is accomplished by comparing the man-made installation and the machine installation. The efficiency of screw mounting is measured via the number of the installed circuit breakers, while the quality of the screw mounting is measured through the degree of deflection of the screw mounting and the preload.The experiment result shows that the efficiency of the screw mounting machine is 12 times higher than that of the manual operation, and the installation quality is significantly higher than the manual installation.

\section{Machine Installation}

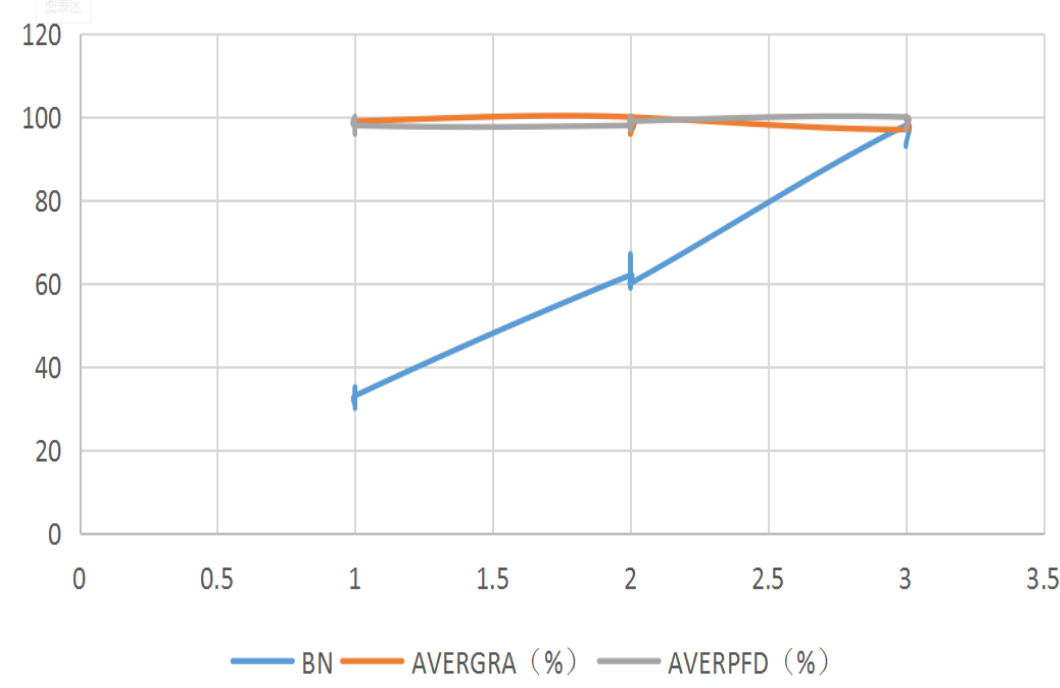

Figure 3 number curve of machine installation

$\mathrm{BN}$-breaker number pretightening force degree
AVERGRA - average value of gradient

AVERPFD - average value of 


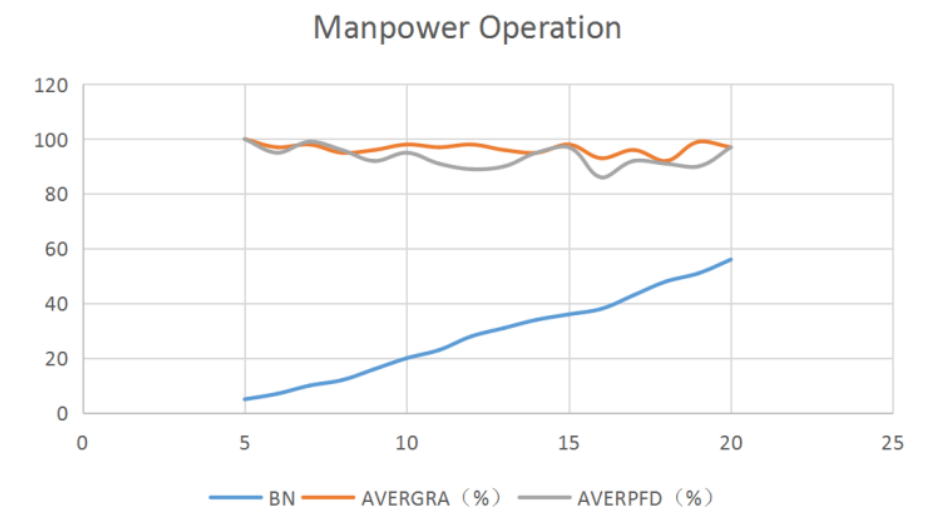

Figure 4 number curve of manpower operation

BN-breaker number

AVERGRA - average value of gradient

AVERPFD - average value of pretightening force degree

The curves of Fig. 3 and Fig. 4 are based on the experimental data, and then calculate through equation (1),(2),(3)and(4).

$$
\begin{aligned}
& \text { PFD }=\frac{\text { M E V }}{\text { PRD V }} \\
& \text { AVERPFD }=\frac{\operatorname{PFD}_{1}+\mathrm{PFD}_{2}+\cdots+\mathrm{PFD}_{n}}{\mathrm{TOTN}^{-}} \\
& G R A=\frac{M E V}{\text { PRD V }} \\
& \mathrm{AVERGRA}=\frac{\mathrm{GRA}_{1}+\mathrm{GRA}_{2}+\cdots+\mathrm{GRA}_{n}}{\text { TOTN }}
\end{aligned}
$$

The experimental efficiency is calculated by the formula (5), (6)and (7).

$$
\begin{aligned}
& \text { AVERPN }=\frac{\text { PNBN }_{1}+\mathrm{PNBN}_{2}+\cdots+\mathrm{PNBN}_{n}}{\mathrm{PN}_{1}+\mathrm{PN}_{2}+\cdots+\mathrm{PN}_{n}}=\frac{458}{200}=2.29 \\
& \text { AVERAM }=\frac{\mathrm{AMNBN}_{1}+\mathrm{AMNBN}_{2}+\cdots+\mathrm{AMNBN}_{n}}{\mathrm{AM}_{1}+\mathrm{AM}_{2}+\cdots+\mathrm{AM}_{n}}=\frac{910}{29}=31.37
\end{aligned}
$$

TRWR $=1-\frac{\text { A VERPON }}{\text { A VERM ON }}=1-\frac{2.29}{31.37}=92.7 \%$

From the calculation,it turns out that the number is 2.29 per worker when it is artificial installation, while it is 31.37 per machine when the machine works. We can concludes finally that the relative efficiency between manual installation and machine installation is merely 7.3.

\section{CONCLUSION}

In the plastic shell breaker screw mounting machine, we put the flexible link, slowdown matching device, stepper motor, electromagnetic clutch integrated together to form a flexible transmission mechanism,this measures could reduce the size of the entire body, and at the same time,prevent the plastic case circuit breaker from being damaged due to the excessive amount of rise. The joining of CCD camera vision positioning system makes complex traditional institutions become simple, while achieves the positioning of micro-components. The flexible biaxial design eliminates the interference movement of the bimetallic plate due to bending deformation during the installation of the screw. Visual positioning and torque sensor may ensure the quality of the installation of screws and the appropriate degree of tightness, and the screw mounting machine can achieve automatic compensation when the circuit breaker metal sheet bends due to temperature and other reasons. The only mobile platform technology makes it more accurate when the screw mounting machine positions in the plane.

\section{REFERENCE}

[1] Si Haili, Cheng Wushan. MCCB intelligent testing system of [J]. low voltage apparatus, 2008, 1 (19): 
34-37.

[2] Han Dongdong. Microcomputer MCCB operating reliability test device detection and control technology [D]. Hebei: Hebei University of Technology, 2007.65-80.[3] Vafiadis, Dimitris ,'Survey Results of Low-voltage Circuit Breakers as Found during

[3] Maintenance Testing: Working Group Report,"IEEE Transactions on Industry Applications. Vol 33,No.4:1367-1369,1997.

[4] Gunhyung Park, Zoran Gajic,,"Sliding mode control of continuous-time weakly coupled linear systems with external disturbances,"AUTOMATICA, Vol.10, pp. 1-5, 2012.

[5] Esteban, Bernat, Riba, Jordi-Roger, Baquero, Grau,"An eddy-current-based sensor for preventing knots in metallic wire drawing processes,"NONDESTRUCTIVE TESTING AND

[6] EVALUATION,Vol.26,pp. 169-180,2011.

[7] Rao, R. Srinivasa, Subbaiah, P. V., Rao, B. Prabhakar,'Electromagnetic transient scattering analysis in time-domain-comparison of TLM and TDIE methods,"JOURNAL OF COMPUTATIONAL

[8] ELECTRONICS,Vol.11,pp.315-320,2012.

[9] Song, SH ,Kang, S, Park, K, “Applications of MEMS-MOSFET Hybrid Switches to Power

[10] Management Circuits for Energy Harvesting Systems,"JOURNAL OF POWER ELECTRONICS,Vol.12,pp. 954-959,2012.

[11] Sheng-Chieh Chen, Chuen-Jinn Tsai,"An axial flow cyclone to remove nanopartic

[12] les at low pressure conditions,"Journal of Nanoparticle Research,vol.9,pp.71-83,2007.

[13] Zhao, Jun, Hill, David J., Liu, Tao,"Stability of dynamical networks with non-identical nodes: A multiple V-Lyapunov function method,"AUTOMATICA, Vol.47, pp.2615-2625, 2011 .

[14] Cheng Wushan. Low voltage electrical appliances intelligent testing technology of [J]. low voltage apparatus, 2010, 12 (2): 11-14.

[15] Liu Weifang. [11] model of linear rolling guide clamp design and research [D]. Wuhan: Huazhong University of Science and Technology, 2011.78-107. 